\title{
Improved model of Delta Orionis
}

\author{
A. Oplištilová ${ }^{1}$, P. Harmanec ${ }^{1}$, P. Mayer ${ }^{1 \dagger}$, P. Zasche ${ }^{1}$, M. Šlechta ${ }^{2}$, \\ H. Pablo ${ }^{3}$, A. Pigulski ${ }^{4}$ and the BRITE Team \\ 1 Astronomical Institute of the Charles University, Faculty of Mathematics \\ and Physics, V Holešovičkách 2, CZ-180 00 Praha 8 - Troja, Czech Republic \\ 2 Astronomical Institute of the Czech Academy of Sciences \\ 25165 Ondřejov, The Czech Republic \\ 3 Département de physique, Université de Montréal, C.P.6128, Succursale \\ center-Ville, Montréal, Québec, H3C 3J7, Canada \\ 4 Instytut Astronomiczny, Uniwersytet Wroctawski, Kopernika 11, 51-622 \\ Wroctaw, Poland
}

Received: September 23, 2019; Accepted: December 17, 2019

\begin{abstract}
We present an improved model of triple star Delta Orionis A. For the first time we were able to disentangle the very weak spectral lines of the secondary in the blue parts of the optical spectrum and derive a reliable mass ratio $q=0.415$. Along with light-curve solutions, based on photometry from the SMEI, MOST and BRITE satellites, we obtained realistic masses and radii of both components of the close binary.
\end{abstract}

Key words: eclipsing binary stars - spectroscopic analysis - light curves

\section{Introduction}

The object $\delta$ Ori A (HD 36486, HIP 25930, HR 1852; $V=2.23$ mag.; average position angle $162.35^{\circ}$ Niesten, 1904) is a triple star in the multiple star system $\delta$ Orionis (Mintaka, ADS 4134) in the constellation of Orion (for the structure of the system see Harvin et al., 2002). The star $\delta$ Orionis A consists of an eclipsing binary with the orbital period of $P=5.732 \mathrm{~d}$ and a distant tertiary with an orbital period of the order of several thousand days.

The binary system has been studied many times. Harvin et al. (2002) carried out a tomographic separation of UV and optical spectra and concluded that the components have unexpectedly low masses: $M_{1}=11.2 \mathcal{M}_{\odot}^{\mathrm{N}}$ and $M_{2}=5.6 \mathcal{M}_{\odot}^{\mathrm{N}}$. Than Mayer et al. (2010) pointed out that the second system of spectral lines in Harvin's study belongs to a tertiary and showed that primary and tertiary dominate the optical spectra. They concluded that the system has normal masses and estimated a mass ratio of about 0.4. Harmanec et al. (2013) indeed reported a similar mass ratio, detecting the secondary in the He I $6678 \AA$ line. A series of detailed studies was published by Corcoran et al. (2015), Nichols et al. (2015), Pablo et al. (2015) and Shenar et al. (2015).

$\dagger$ Pavel Mayer passed away on the day of his $86^{\text {th }}$ birthday Nov. 7, 2018 


\section{Observational material used and data analysis}

All electronic spectra covering the blue and green spectral region (RJD between 50031 and 58405) obtained at the Ondřejov 2-m reflector were used. These were complemented by spectra from the Haute Provence Observatory Elodie echelle spectrograph and the ESO LaSilla Feros echelle spectrograph. The space-based photometric data was obtained with instruments on board SMEI, MOST and BRITE $^{1}$ (RJD between 52676 and 56995).

Normalization of spectra, removal of residual cosmic rays and radial-velocity (RV) measurements were carried out with the program SPEFO (Horn et al., 1996; Škoda, 1996), developed by Mr. J. Krpata (Krpata, 2008). We disentangled the spectra in KOREL (Hadrava, 2004) in two steps: we first disentangled the strong spectra of the primary and tertiary and then we disentangled the spectrum of the faint secondary in the residual spectra from the first step. They were then fitted by interpolated synthetic spectra with the help of the program PYTERPOL ${ }^{2}$ to fit the profiles of the components (see Fig. 1). For the final combined RV and light-curve solutions we used the program PHOEBE $1^{3}$ (Prša \& Zwitter, 2005).

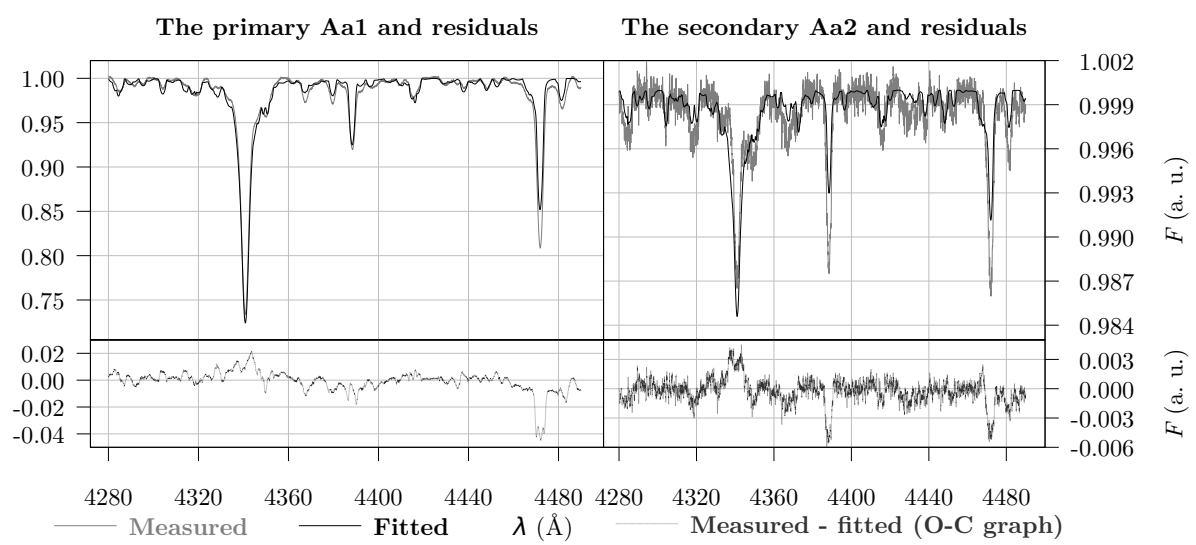

Figure 1. Comparison of disentangled spectra of the primary and secondary with the best-fit synthetic spectra found by PYTERPOL.

\footnotetext{
${ }^{1}$ Based on data collected by the BRITE Constellation satellite mission, designed, built, launched, operated and supported by the Austrian Research Promotion Agency (FFG), the University of Vienna, the Technical University of Graz, the University of Innsbruck, the Canadian Space Agency (CSA), the University of Toronto Institute for Aerospace Studies (UTIAS), the Foundation for Polish Science \& Technology (FNiTP MNiSW), and National Science Centre $(\mathrm{NCN})$

${ }^{2}$ https://github.com/chrysante87/pyterpol/wiki

${ }^{3}$ http://phoebe-project.org/1.0
} 


\section{Results}

The final elements are in Table 1. It was found that the solution based on the $S M E I$ data led to a high inclination and an anomalously small radius for the secondary. A realistic solution was found with the BRITE and MOST photometry together. Fig. 2 displays the fitted light curves. Using $S M E I$ we arrived at reasonable masses and radii for both the primary (cf. Martins et al., 2005) and secondary (see Harmanec, 1988).

Table 1. Solution

\begin{tabular}{lcc}
\hline \hline Parameters & \multicolumn{1}{c}{ BRITE } & \multicolumn{1}{c}{ SMEI } \\
\hline$a / \mathcal{R}_{\odot}^{\mathrm{N}}$ & $41.91 \pm 0.18$ & $40.71 \pm 0.21$ \\
$\omega /{ }^{\circ}$ & $148.73 \pm 1.49$ & $158.37 \pm 0.71$ \\
$\gamma / \mathrm{km} \mathrm{s}^{-1}$ & $21.96 \pm 0.33$ & $22.28 \pm 0.41$ \\
$i /^{\circ}$ & $78.1 \pm 0.3$ & $91.6 \pm 0.4$ \\
\hline$M_{1} / \mathcal{M}_{\odot}^{\mathrm{N}}$ & 21.1 & 19.4 \\
$M_{2} / \mathcal{M}_{\odot}^{\mathrm{N}}$ & 8.8 & 8.1 \\
$R_{1} / \mathcal{R}_{\odot}^{\mathrm{N}}$ & 13.6 & 10.4 \\
$R_{2} / \mathcal{R}_{\odot}^{\mathrm{N}}$ & 3.7 & 1.71 \\
$M_{\mathrm{bol}_{1}} / \mathrm{mag}$ & -8.28 & -7.69 \\
$M_{\mathrm{bol}_{2}} / \mathrm{mag}$ & -4.55 & -2.87 \\
$L_{\mathrm{R}_{1}}$ & 0.690 & 0.712 \\
$L_{\mathrm{R}_{2}}$ & 0.037 & 0.014 \\
$\log _{10} g_{1}$ & 3.50 & 3.70 \\
$\log _{10} g_{2}$ & 4.24 & 4.88 \\
$\chi_{\mathrm{N}}^{2}$ & 11.389 & 1.008 \\
\hline \hline
\end{tabular}

\begin{tabular}{ll}
\hline \hline Fixed param. & Values \\
\hline$P / \mathrm{d}$ & $5.732436^{*}$ \\
$\dot{\omega} /{ }^{\circ} \mathrm{d}^{-1}$ & $0.004220^{*}$ \\
$q=M_{2} / M_{1}$ & $0.41549^{* *}$ \\
$e$ & $0.07583^{* *}$ \\
$T_{\text {eff }_{1}} / \mathrm{K}$ & $31401^{* * *}$ \\
$T_{\text {eff }_{2}} / \mathrm{K}$ & $25442^{* * *}$ \\
$L_{\mathrm{R}_{3}}$ & $0.273^{* * *}$ \\
\hline
\end{tabular}

* Mayer et al. (2010)

** from KOREL

*** from PYTERPOL

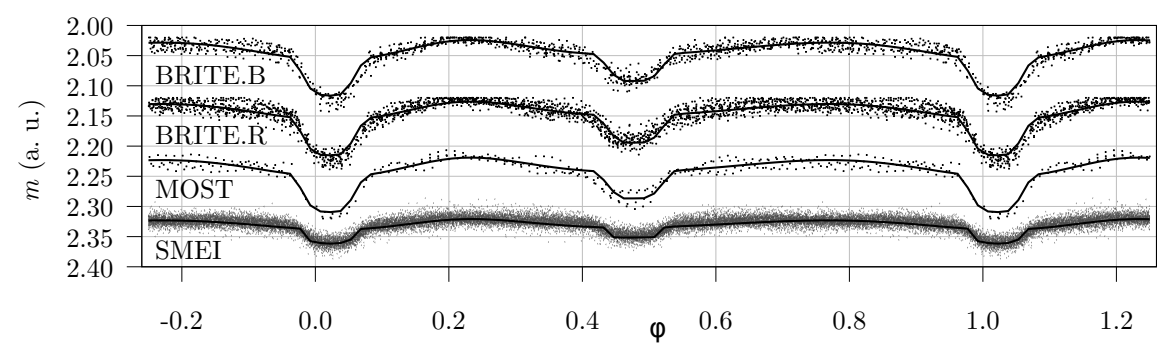

Figure 2. Fitted light curves (from the program PHOEBE) 
Acknowledgements. We acknowledge the use of the programs KOREL by Petr Hadrava, PHOEBE 1 by Andrej Prša, and PYTERPOL, and some auxiliary programs by J. Nemravová. Our thanks are also due to Š. Dvořáková, D. Korčáková, J. Kubát, J. Nemravová, M. Oksala, P. Škoda, and V. Votruba who obtained some of the Ondřejov blue spectra used in this study. This research was supported by the grants P209/10/0715, GA15-02112S, and GA19-01995S of the Czech Science Foundation.

\section{References}

Corcoran, M. F., Nichols, J. S., Pablo, H., et al., A Coordinated X-Ray and Optical Campaign of the Nearest Massive Eclipsing Binary, $\delta$ Orionis Aa. I. Overview of the X-Ray Spectrum. 2015, Astrophys. J., 809, 132

Hadrava, P., KOREL - User's guide. 2004, Publ. Astron. Inst. Acad. Sci. Czech Rep., 92, 15

Harmanec, P., Stellar Masses and Radii Based on Modern Binary Data. 1988, Bulletin of the Astronomical Institutes of Czechoslovakia, 39, 329

Harmanec, P., Mayer, P., \& Šlechta, M., The Massive Binary Delta Ori and the Problem of the Spectroscopic Detection of its Weak Secondary. 2013, in Massive Stars: From alpha to Omega, 70

Harvin, J. A., Gies, D. R., Bagnuolo, William G., J., Penny, L. R., \& Thaller, M. L., Tomographic Separation of Composite Spectra. VIII. The Physical Properties of the Massive Compact Binary in the Triple Star System HD 36486 ( $\delta$ Orionis A). 2002, Astrophys. J., 565, 1216, DOI: 10.1086/324705

Horn, J., Kubát, J., Harmanec, P., et al., Spectroscopic orbit of the triple star 55 Ursae Majoris. 1996, Astron. Astrophys., 309, 521

Krpata, J. 2008, http://astro.troja.mff.cuni.cz/ftp/hec/SPEFO/

Martins, F., Schaerer, D., \& Hillier, D. J., A new calibration of stellar parameters of Galactic O stars. 2005, Astron. Astrophys., 436, 1049

Mayer, P., Harmanec, P., Wolf, M., Božić, H., \& Šlechta, M., Physical elements of the eclipsing binary $\delta$ Orionis. 2010, Astron. Astrophys., 520, A89

Nichols, J., Huenemoerder, D. P., Corcoran, M. F., et al., A Coordinated X-Ray and Optical Campaign of the Nearest Massive Eclipsing Binary, $\delta$ Orionis Aa. II. X-Ray Variability. 2015, Astrophys. J., 809, 133, DOI: 10.1088/0004-637X/809/2/133

Niesten, L., Mesures micromtriques d'toiles doubles faites Bruxelles et Uccle de 1878 1896. 1904, Annales de l'Observatoire royal de Belgique Nouvelle serie

Pablo, H., Richardson, N. D., Moffat, A. F. J., et al., A Coordinated X-Ray and Optical Campaign of the Nearest Massive Eclipsing Binary, $\delta$ Orionis Aa. III. Analysis of Optical Photometric (MOST) and Spectroscopic (Ground-based) Variations. 2015, Astrophys. J., 809, 134, DOI: 10.1088/0004-637X/809/2/134

Prša, A. \& Zwitter, T., A Computational Guide to Physics of Eclipsing Binaries. I. Demonstrations and Perspectives. 2005, Astrophys. J., 628, 426, DOI: $10.1086 / 430591$ 
Shenar, T., Oskinova, L., Hamann, W. R., et al., A Coordinated X-Ray and Optical Campaign of the Nearest Massive Eclipsing Binary, $\delta$ Orionis Aa. IV. A Multiwavelength, Non-LTE Spectroscopic Analysis. 2015, Astrophys. J., 809, 135

Škoda, P., SPEFO - A Simple, Yet Powerful Program for One-Dimensional Spectra Processing. 1996, in ASP Conf. Ser. 101: Astronomical Data Analysis Software and Systems V, 187-189 